\title{
Different patterns of pelvic ureteral endometriosis. What is the best treatment? Results of a retrospective analysis
}

\author{
Salvatore Butticè ${ }^{1}$, Antonio Simone Laganà ${ }^{2}$, Giuseppe Mucciardi ${ }^{1}$, Francesco Marson ${ }^{3}$, Tzevat Tefik ${ }^{4}$, \\ Christopher Netsch ${ }^{5}$, Salvatore Giovanni Vitale ${ }^{2}$, Emre Sener ${ }^{6}$, Rosa Pappalardo ${ }^{1}$, Carlo Magno ${ }^{1}$ \\ ${ }^{1}$ Department of Human Phatology, Section of Urology, University of Messina, Messina, Italy; \\ ${ }^{2}$ Unit of Gynecology and Obstetrics, Department of Human Pathology in Adulthood and Childhood "G. Barresi", University of \\ Messina, Messina, Italy; \\ ${ }^{3}$ Department of Urology, University of Studies of Torino, Torino, Italy: \\ ${ }^{4}$ Department of Urology, Istanbul University, Istanbul Faculty of Medicine, Istanbul, Turkey: \\ ${ }^{5}$ Department of Urology, Asklepios Hospital Barmbek, Hamburg, Germany: \\ ${ }^{6}$ Department of Urology, School of Medicine, Marmara University, Istanbul, Turkey.
}

\begin{abstract}
Summary Objective. Endometriosis is an estrogendependent disease. The incidence of urinary tract endometriosis (UE) increased during the last few years and, nowadays, it ranges from 0.3 to $12 \%$ of all women affected by the disease. The ureter is the second most common site affected. The ureteral endometriosis is classified in extrinsic and intrinsic. The aim of this study is to individuate the best treatments for each subset of ureteral endometriosis. Materials and Methods. 32 patients diagnosed with surgically treated UE were retrospectively reviewed. The patients were divided into 3 subsets (intrinsic UE, extrinsic UE with and without obstruction). The patients with intrinsic UE $(n=10)$ were treated with laser endoureterotomy. The patients with extrinsic UE $(n=22)$ were divided in two subsets with ( $n$ $=16)$ and without $(n=6)$ hydronephrosis. All the patients underwent ureteral stenting, and resection and reimplantation was performed in the first group, and when the mass was $>2.5 \mathrm{~cm}(n=3)$ Boari flap was performed. Laparoscopic ureterolysis (shaving) was performed in the second group. Results. In the extrinsic subset of UE, we obtained an high therapeutic success (84\%). Conversely, in the intrinsic subset there was a recurrence rate of the disease in $6 / 10$ of the patients $(60 \%)$.

Conclusions. Ureterolysis seems to be a good treatment in extrinsic UE without obstruction. Resection and reimplantation allows excellent results in the extrinsic UE with obstruction. In the intrinsic subset, the endoureterotomy approach is inadequate.
\end{abstract}

KEY WORDS: Ureteral endometriosis; Laparoscopic ureterolysis; Laser endoureterotomy; Ureteral reimplantation; Laparoscopy.

Submitted 3 April 2016; Accepted 22 May 2016

\section{INTRODUCTION}

Endometriosis is an estrogen-dependent disease (1) characterized by the ectopic presence and growth of functional endometrial tissue, glands and stroma, outside the uterine cavity $(2,3)$.

Approximately $10 \%$ of women in reproductive age are estimated to be affected by this disease $(4,5)$. Its symptoms and signs include acute or chronic pelvic pain, abnormal bleeding, infertility (6).
The disease most often affects the ovaries (up to $88 \%$ of all cases), sacrouterine ligaments, fallopian tubes, rectum, cervico-vaginal zone, and urinary tract.

The incidence of urinary tract endometriosis (UTE) increased during the last few years and, nowadays, it ranges from 0.3 to $12 \%$ of all women affected by the disease (7). The ureter is the second most common site affected by UTE, after bladder, showing an increasing incidence in the contemporary literature because of the improvement in diagnostic tools as well as a greater awareness and skill among clinicians in recognizing this disease. In most cases ureteral involvement is completely unnoticed $(8,9)$. There are two major types of ureteral endometriosis (UE): intrinsic and extrinsic, occurring respectively with a 1:4 ratio (10-12).

The distinction of the extrinsic or intrinsic nature of ureteral involvement may be considered as arbitrary. The depth of invasion in the extrinsic form has to be confirmed by the final histological examination; thus, differentiation cannot be reliably made, either preoperatively or during surgery. In extrinsic pattern, the endometrial tissue invades only the ureteral adventitia or surrounding connective tissue and, consequently, it causes ureteral obstruction (13-15). This is the most common form of UE and represents $80 \%$ of cases (16). In intrinsic disease (which occurs in only $20 \%$ of cases), ectopic endometrial tissue directly infiltrates the muscle layer of the ureteral wall within the muscularis propria, lamina propria or ureteral lumen; nevertheless, the two types of UE may coexist. The preoperative diagnosis and choice of an appropriate surgical approach are both essential for the treatment of patients with ureteral endometriosis.

Regarding surgical treatment for ureteral endometriosis, currently the gold standard is represented by laparoscopy (17), although others (18) suggest the use of robotic surgery in order to improve surgical accuracy and minimize complications.

Considering this evidence, the purpose of our study was to identify the best surgical treatments for different types of UE. 


\section{Materials AND MEthods}

As standard protocol of the university hospitals in which the study was carried out, each patient was informed at the admission and signed an informed consent allowing data collection for research purposes. The study design is in accordance with the Helsinki Declaration, conforms the Committee on Publication Ethics (COPE) guidelines and was approved by the Institutional Review Board (IRB) of the university hospitals in which it was performed. All the design, analysis, interpretation of data, drafting and revisions followed the Strengthening the Reporting of Observational Studies in Epidemiology (STROBE) Statement: guidelines for reporting observational studies (19), available through the EQUATOR (Enhancing the Quality and Transparency of Health Research) network. Below the surgical approaches that were used to treat different types of UE are described. We enrolled only the patients with histologically confirmed endometriosis.

\section{Management of the intrinsic ureteral endometriosis}

Accumulating evidence suggests that indications for endoureterotomy include an intrinsic ureteral lesion and/or lesions smaller than $3 \mathrm{~cm}$ with the lesion being located under the level of the iliac vessels $(14,20)$. In our case series, we used a 0.035 inch guidewire to introduce the ureteroscope through the ureteral orifice. The Storz rigid ureteroscope was applied in all cases. Moreover, we performed cup biopsy of the ureteral mass, and a $6.0 \mathrm{~F}$ stent was left in each patient. One week later, after the histological confirmation of ureteral endometriosis, patients underwent an endoureterotomy with laser (120W 2- $\mu$ m continuous-wave Tm:YAG laser, Revolix 2). A retrograde cutting pattern was performed, by slowly advancing the ureteroscope proximally. The incision began at roughly $0.5 \mathrm{~cm}$ below the mass margin and extended over the obstruction for another $0.5 \mathrm{~cm}$. The ureteroscope could be used to assist the procedure by mild lateral parting of the cutting edges. In case of bleeding, we cauterized the lesion by defocused laser beam and the incision was deepened until the periureteral fat was seen. A 6 F stent was left indwelling for 4 weeks with urethral catheterization of 2 days.

\section{Management of the extrinsic ureteral endometriosis}

The enrolled cases of extrinsic UE were divided in in two subsets: with or without obstructive pattern. All patients of the two subsets underwent pre-surgical preparation, began 24 hours prior to surgery with oral intake of SelgEsse 1000 (Promefarm, Milan, Italy), plus Mylicon tablets (Warner Lambert, Milan, Italy). Ureteral stenting (6 F) was performed before the procedure. All patients underwent laparoscopic approach with standard technique including the use of 10-mm operative laparoscope and three 5-mm ancillary trocars, at $12-\mathrm{mm} \mathrm{Hg}$ intra-abdominal pressure. The first surgical step was the same for each subset, consisting in the exposure of the peritoneum and careful blunt dissection of the ureter. In cases without obstructive pattern, the dissection started where the ureter was clearly visible and without adhesions, and progressed in the direction of the uterosacral ligaments until insertion into the bladder. At the end of the dissection, the ureter was completely mobilized and visible from the pelvic brim to its insertion into the bladder. Endometriosis lesions on the ureter, both those circularly encasing it and those involving just part of its circumference, were carefully removed. In cases with obstructive pattern, after ureteral mobilization, we performed resection and reimplantation (LichGregoire technique); moreover, in 3 of the 16 patients, because of the extension of the mass $(>2.5 \mathrm{~cm})$, we had to perform also a Boari flap to obtain a tension-free anastomosis. At follow-up, we performed ultrasound after one week, urography, ultrasound, blood (included CA125) and urine tests after one, six months and one year Recurrence of the disease was defined by ultrasound, urography and serum level of CA125.

\section{RESULTS}

We performed a retrospective analysis of our data, collected from March 2000 to August 2014. We enrolled 32 patients, 10 with intrinsic and 22 with extrinsic UE. In the extrinsic subset, we enrolled 16 patients with and 6 patients without obstructive pattern. The clinic characteristics and the symptoms associated of the patients are shown in the Tables 1 and 2 . All the operations were performed by two surgeons with great experience in endourology, open and laparoscopic surgery. In the

\section{Table 1.}

Patients characteristics.

\begin{tabular}{|lccc|}
\hline & $\begin{array}{c}\text { Extrinsic UE } \\
\text { obstructive } \\
(\mathbf{n}=16)\end{array}$ & $\begin{array}{c}\text { Extrinsic UE } \\
\text { without obstruction } \\
(\mathbf{n}=\mathbf{6})\end{array}$ & $\begin{array}{c}\text { Intrinsic UE } \\
(\mathbf{n}=\mathbf{1 0})\end{array}$ \\
\hline Age (Mean \pm SD) & $42.18 \pm 11.25$ & $42.83 \pm 10.10$ & $47.6 \pm 10.75$ \\
\hline BMI (Mean \pm SD) & $23.68 \pm 4.81$ & $20.83 \pm 3.92$ & $23.6 \pm 4.85$ \\
\hline Site of involvment & & & \\
Left $(n, \%)$ & $10(62.5)$ & $4(66.6)$ & $5(50)$ \\
Right (n,\%) & $5(31.2)$ & $2(33.3)$ & $5(50)$ \\
Bilateral (n,\%) & $1(6.2)$ & 0 & 0 \\
\hline Comorbidities & & & \\
Hypertension (n,\%) & $4(25)$ & $1(16.6)$ & $3(30)$ \\
Diabetes (n,\%) & $1(6.2)$ & 0 & $2(20)$ \\
Metabolic syndrome (n,\%) & $2(12.5)$ & 0 & $1(10)$ \\
Hypercholesterolemia (n,\%) & 0 & $1(16.6)$ & 0 \\
PCOS (n,\%) & $3(18.7)$ & $2(33.3)$ & 0 \\
& & & \\
\hline
\end{tabular}

Table 2.

Symptoms in patients with ureteral endometriosis.

\begin{tabular}{|lccc|}
\hline & $\begin{array}{c}\text { Extrinsic UE } \\
\text { obstructive } \\
(\mathbf{n}=\mathbf{1 6})\end{array}$ & $\begin{array}{c}\text { Extrinsic UE } \\
\text { without obstruction } \\
(\mathbf{n}=\mathbf{6})\end{array}$ & $\begin{array}{c}\text { Intrinsic UE } \\
(\mathbf{n}=\mathbf{1 0})\end{array}$ \\
\hline Dysmenorrhea $(\mathrm{n}, \%)$ & $7(43.7)$ & $1(16.6)$ & $3(30)$ \\
\hline Dyspareunia(n,\%) & $4(25)$ & 0 & $2(20)$ \\
\hline Chronic Pelvic pain (n,\%) & $2(12.5)$ & $1(16.6)$ & $1(10)$ \\
\hline Hematuria $(n, \%)$ & $7(43.7)$ & $3(50)$ & $5(50)$ \\
\hline Recurrent UTI $(n, \%)$ & $3(18.7)$ & $2(33.3)$ & $3(30)$ \\
\hline Infertility $(n, \%)$ & $6(37.5)$ & $2(33.3)$ & 0 \\
\hline LUTS $(n, \%)$ & $2(12.5)$ & $1(16.6)$ & $4(40)$ \\
\hline No symptoms $(n, \%)$ & $1(6.2)$ & $2(33.3)$ & 0 \\
\hline
\end{tabular}


Figure 1.

Graphic recurrence probability/time (months).

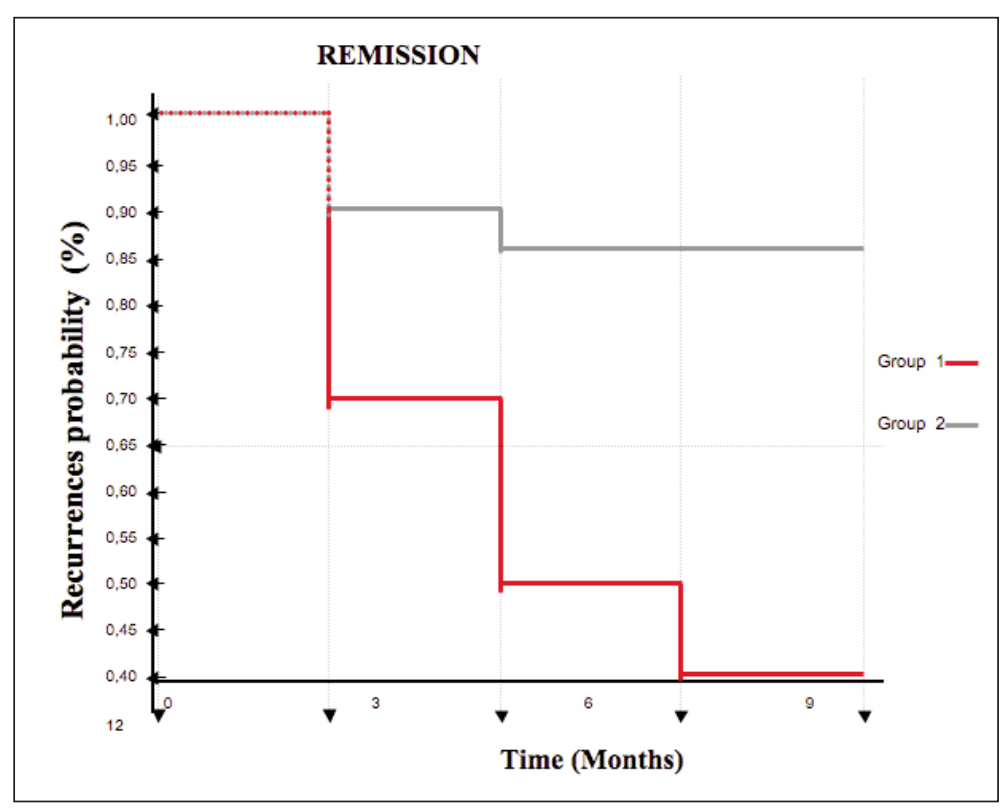

assumptions, to date surgery still maintains a key role in the management of UE. The aim of surgery is to remove or relieve obstruction, especially in cases of advanced stage of disease, and to resolve the haematuria. In III-IV stages, in fact, there is a predominance of profibrotic response (5) and the derived fibrotic perilesional tissue, typically, does not respond to hormonal therapy (26-28). Despite numerous papers previously published in the literature, to date there is no definitive answer about the best surgical procedure to manage UE. It is widely accepted that two surgical approaches are mainly used: resection of the ureteral segment and ureterolysis (which frees the ureter from extrinsic compression through a laparoscopic "shaving" technique). Most surgeons are familiar with both techniques, though the reasons that guide their choice remain unclear, particularly as recommendations on the surgical management of UTE tend to reflect experience or personal convictions based on scarce evidence. Thus, some authors (29) recommend radical resection to reduce the risk of recurrences as much as possible,

extrinsic subset of UE, we obtained a good urological therapeutic success (84\%). Conversely, in the intrinsic subset there was a recurrence of the disease, in 6 patients (60\%) grade 1 hydronephrosis persisted with one of the patients having haematuria. In the extrinsic UE, 10 patients underwent to open surgery and 12 to laparoscopic surgery. No major complications occurred, although two patients treated with open surgery had surgical wound infection and another one needed blood transfusion.

Kaplan-Meier survival curve shows (Figure 1) the difference between two groups; comparison of recurrence curves was made by using log-rank test, which was statistically significant $(\mathrm{p}=0.0007)$.

\section{Discussion}

The goal of treatment for UE is to relieve obstruction and preserve as much renal function as possible. In most cases, management needs multi-disciplinary approach (21). Medical therapy is indicated only for early-stage disease, but not for advanced disease due to high recurrence and the limited effect on scar tissue. According to Zanetta et al. (23), medical treatment after total abdominal hysterectomy with bilateral salpingo-oophorectomy can decrease the recurrence from $27 \%$ to $3 \%$. Of course, this combined treatment is not advisable when the patient is young and it is mandatory to preserve its fertility. Furthermore, there is no consensus on which medical treatment is the best to manage UE, currently danazol, GnRH agonist (leuprolide, goserelin), medroxyprogesterone, oestrogen-progestin combination and progestin alone being used $(23,24)$. In particular, danazol and GnRH are most commonly used in case of III-IV stages, with the aim of antagonizing the effect of gonadotropin, entailing the eventual effect of inhibition of ovarian function. Even if several authors reported use of these two hormonal treatments, clearly evidence about long-term outcome is still missing (25). Considering these while others (30) reserve ureterectomy only for limited cases and believe conservative surgery to be the management of choice in relieving ureteral obstruction and removing endometriosis mass, even in patients with moderate or severe pyelic dilatation. The "carcinologic" approach is adopted by surgeons who strongly believe that the radical removal of all endometriotic foci is the most effective way to prevent the risk of recurrences, much as radical surgery does in cancer. Thus, ureteral resection should be performed to avoid leaving intrinsic implants. Conversely, other authors proposed an approach to UTE that focuses more on the woman's needs and the resolution of symptoms, than on prior lesion excision. This approach becomes possible where medical treatment is associated with surgery and long-term administered. In our experience regarding extrinsic subset, we performed a radical approach in cases with obstructive pattern and a conservative one in cases without obstructive pattern: this seems to be effective and consent to minimize side effects maximizing the results. Regarding intrinsic subset (always obstructive), we performed an endoscopic approach (endoureterotomy) with laser: this has been proven to be effective only in $40 \%$ of cases, making it a controversial option.

\section{Conclusion}

Despite our study is limited by the little number of patients and the small period of follow-up, we can suggest that in the extrinsic UE subset without obstructive pattern, ureterolysis is an appropriate treatment. In the extrinsic UE with obstructive subset, the resection and reimplantation allows excellent results. In the intrinsic subset, the endoureterotomy approach reported high rates of failure, showing to be effective only in $40 \%$ of patients. This finding suggests the general idea that, in case of intrinsic UE, endoscopic treatment can be proposed by advising the patients that the rate of success is only $40 \%$; even if our 
number of treated patients is relatively small, our KaplanMeier curves clearly shows that recurrence generally appears within one year after surgery: this suggest to plan an early follow-up, in order to detect recurrences as early as possible. A second possibility could be to treat intrinsic UE by resection and reimplantation from the beginning; this is also a viable possibility, considering low rates of success by endourological approach.

Further evaluations with a more consistent number of patients and with longer follow-up are still needed before solid conclusions can be drawn.

\section{REFERENCES}

1. Giudice LC, Kao LC. Endometriosis. Lancet. 2004; 364:1789-99.

2. Laganà AS, Sturlese E, Retto $G$, et al. Interplay between Misplaced Müllerian-Derived Stem Cells and Peritoneal Immune Dysregulation in the Pathogenesis of Endometriosis. Obstet Gynecol Int. 2013; 2013:527041.

\section{Bulun SE. Endometriosis. N Engl J Med. 2009; 360:268-279.}

4. Sofo V, Götte M, Laganà AS, et al. Correlation between dioxin and endometriosis: an epigenetic route to unravel the pathogenesis of the disease. Arch Gynecol Obstet. 2015; 292:973-86.

5. Marana R, Lecca A, Biscione, et al. Endometriosis: the gynecologist's opinion. Urologia. 2012; 79:160-166.

6. Triolo O, Laganà AS, Sturlese E. Chronic pelvic pain in endometriosis: an overview. J Clin Med Res. 2013; 5:153-63.

7. Maccagnano C, Pellucchi F, Rocchini L, et al. Ureteral endometriosis: proposal for a diagnostic and therapeutic algorithm with a review of the literature. Urol Int. 2013; 91:1-9.

8. Nezhat C, Nezhat F, Nezhat CH, et al. DS: Urinary tract endometriosis treated by laparoscopy. Fertil Steril 1996; 66:920-924.

9. Butticè S, Laganà AS, Barresi V, et al. Lumbar Ureteral Stenosis due to Endometriosis: Our Experience and Review of the Literature. Case Rep Urol. 2013; 2013:812475.

10. Mounsey AL, Wilgus A, Slawson DC. Diagnosis and management of endometriosis. Am Fam Physician 2006; 74:594-600.

11. Mahutte NG, Arici A. Medical management of endometriosisassociated pain. Obstet Gynecol Clin North Am. 2003; 30:133-150.

12. Takagi H, Matsunami K, Ichigo S, et al. Novel medical management of primary bladder endometriosis with dienogest: a case report. Clin Exp Obstet Gynecol. 2011; 38:184-185.

13. Comiter CV. Endometriosis of the urinary tract. Urol Clin North Am 2002;29:625-635

14) Yohannes P: Ureteral endometriosis. J Urol. 2003; 170:20-25.

15. Seracchioli R, Mabrouk M, Montanari G, et al. Conservative laparoscopic management of urinary tract endometriosis (UTE): surgical outcome and long-term follow-up. Fertil Steril. 2010; 94:856-861.

16. Takamura M, Koga K, Osuga Y, et al. Post-operative oral contraceptive use reduces the risk of ovarian endometrioma recurrence after laparoscopic excision. Hum Reprod. 2009; 24:3042-3048.

17. Antonelli A. Urinary tract endometriosis. Urologia. 2012; 79:167-70.

18. Nezhat C, Modest AM, et al. The role of the robot in treating urinary tract endometriosis. Curr Opin Obstet Gynecol. 2013; 25:308-11.

19. Von Elm E, Altman DG, Egger M, et al. The Strengthening the Reporting of Observational Studies in Epidemiology (STROBE) statement: guidelines for reporting observational studies. Lancet. 2007; 370:1453-1457.

20. Marcelli F, Collinet P, Vinatier D, et al. Ureteric and bladder involvement of deep pelvic endometriosis. Value of multidisciplinary surgical management. Prog Urol. 2006; 16:588-593.

21. Langebrekke A, Qvigstad E. Ureteral endometriosis and loss of renal function: mechanisms and interpretations. Acta Obstet Gynecol Scand. 2011; 90:1164-6.

22- Takeuchi S, Minoura H, Toyoda N, et al. Intrinsic ureteric involvement by endometriosis: a case report. J Obstet Gynaecol Res. 1997; 23:273

23. Zanetta G, Webb M, Segura JW. Ureteral endometriosis diagnosed at ureteroscopy. Obstet Gynecol. 1998; 91:857.

24. Pittaway DE, Daniell JF, Maxson WS, et al. Recurrence of ureteral obstruction caused by endometriosis after danazol therapy. Am J Obstet Gynecol. 1982; 143:720.

25. Matsuura K, Kawasaki N, Oka M, et al. Treatment with danazol of ureteral obstruction caused by endometriosis. Acta Obstet Gynecol Scand. 1985; 64:339.

26. Feng CC, Ding Q, Zhang YF, et al. Pigment epithelium-derived factor expression is down-regulated in bladder tumors and correlates with vascular endothelial growth factor and matrix metalloproteinase-9. Int Urol Nephrol. 2011; 43:383-90.

27- El Kahder K, Guille F, Patard JJ, et al. Ureteral reimplantation on psoas bladder: long-term results. Acta Urol Belg. 1998; 66:15.

28. Kupajski M, Tkocz M, Ziaja D. Modern management of stone disease in patients with a solitary kidney. Videosurgery Miniinv. 2012; 7:1-7.

29. Chapron C, Chiodo I, Leconte $M$, et al. Severe ureteral endometriosis: the intrinsic type is not so rare after complete surgical exeresis of deep endometriotic lesions. Fertil Steril. 2010; 93:21152120.

30. Donnez J, Nisolle M, Squifflet J. Ureteral endometriosis: a complication of rectovaginal endometriotic (adenomyotic) nodules. Fertil Steril. 2002; 77:32-37.

\section{Correspondence}

Salvatore Butticè, MD (Corresponding Author)

salvobu@gmail.com

Giuseppe Mucciardi, MD

Rosa Pappalardo, MD

Carlo Magno, MD

Department of Human Phatology, Section of Urology,

University of Messina

Via Consolare Valeria 1, 98125 Messina, Italy

Antonio Simone Laganà, MD

Salvatore Giovanni Vitale, MD

Unit of Gynecology and Obstetrics, Department of Human Pathology in Adulthood and Childhood "G. Barresi", University of Messina,

Messina, Italy

Francesco Marson, MD

Department of Urology, University of Studies of Torino, Torino, Italy

Tzevat Tefik, MD

Istanbul University, Istanbul Faculty of Medicine, Department of Urology, Istanbul, Turkey

\section{Christopher Netsch, MD}

Department of Urology, Asklepios Hospital Barmbek, Hamburg, Germany

Emre Sener, MD

Department of Urology, School of Medicine, Marmara University, Istanbul, Turkey 